\title{
Plains zebra (Equus quagga) adrenocortical activity increases during times of large aggregations in the Serengeti ecosystem
}

\author{
Seeber $P A^{1}$, Franz $M^{1}$, Dehnhard $M^{1}$, Ganswindt $A^{2,3}$, Greenwood $A D^{1,4, \#, *}$ \& East $\mathrm{ML}^{1, \#}$ \\ ${ }^{1}$ Leibniz-Institute for Zoo and Wildlife Research, Alfred-Kowalke-Strasse 17, 10315 Berlin, Germany \\ ${ }^{2}$ Endocrine Research Laboratory, Department of Anatomy and Physiology, Faculty of Veterinary \\ Science, University of Pretoria, Private Bag XO4, Onderstepoort 0110, South Africa \\ ${ }^{3}$ Mammal Research Institute, Department of Zoology and Entomology, Faculty of Natural and \\ Agricultural Sciences, University of Pretoria, Pretoria 0028, South Africa \\ ${ }^{4}$ Department of Veterinary Medicine, Freie Universität Berlin, Berlin, Germany \\ \# equal contribution \\ *corresponding author: greenwood@izw-berlin.de
}

\begin{abstract}
Adverse environmental stimuli (stressors) activate the hypothalamic-pituitary-adrenal (HPA) axis and contribute to allostatic load. This study investigates the contribution of environmental stressors and life history stage to allostatic load in a migratory population of plains zebras (Equus quagga) in the Serengeti ecosystem, in Tanzania, which experiences large local variations in aggregation. We expected higher fGCM response to the environmental stressors of feeding competition, predation pressure and unpredictable social relationships in larger than in smaller aggregations, and in animals at energetically costly life history stages. As the study was conducted during the $2016 \mathrm{El} \mathrm{Niño,} \mathrm{we} \mathrm{did}$ not expect food quality of forage or a lack of water to strongly affect $\mathrm{fGCM}$ responses in the dry season. We measured fecal glucocorticoid metabolite (fGCM) concentrations using an enzyme immunoassay (EIA) targeting 11ß-hydroxyetiocholanolone and validated its reliability in captive plains zebras. Our results revealed significantly higher fGCM concentrations 1 ) in large aggregations than in smaller groupings, and 2) in band stallions than in bachelor males. Concentrations of fGCM were not significantly higher in females at the energetically costly life stage of late pregnancy/lactation. The higher allostatic load of stallions associated with females, than bachelor males is likely caused by social stressors. In conclusion, migratory zebras have elevated allostatic loads in large aggregations that probably result from their combined responses to increased feeding competition, predation pressure and various social stressors. Further research is required to disentangle the contribution of these stressors to allostatic load in migratory populations.
\end{abstract}

Keywords: plains zebra, Serengeti, glucocorticoids, aggregation, allostatic load, non-invasive hormone monitoring

Highlights

- Validation of an assay for fecal glucocorticoid metabolite measurement in E. quagga

- Allostatic load of plains zebras is higher in large aggregations

- Band stallions have significantly higher fecal glucocorticoid levels than bachelors

- Lactation and late pregnancy did not affect allostatic load 


\section{Introduction}

In vertebrates, the hypothalamic-pituitary-adrenal (HPA) axis is the neuroendocrine pathway by which glucocorticoids are released from the adrenal cortex in response to adverse environmental stimuli termed stressors (Creel et al., 2013; Hofer and East, 2012; Nelson et al., 2002). Glucocorticoids influence the expression of genes and gene pathways with diverse functions including those that mobilize energy required for behavioral and physiological responses to stressors (Phuc Le et al., 2005; Tung et al., 2012). The term allostatic load encompasses the cumulative energetic cost of maintaining homeostasis, fulfilling the physiological costs of daily life that vary with life history stage (Stearns, 1992), and costs of responding to environmental stressors (McEwen and Wingfield, 2003; Romero et al., 2009). Glucocorticoid (GC) concentrations are considered a useful indicator of allostatic load (Goymann and Wingfield, 2004; McEwen and Wingfield, 2003) and a proxy measure of the contribution of environmental stressors to allostatic load (e.g. Benhaiem et al., 2013; Clinchy et al., 2013; Nelson et al., 2002; Rubenstein and Shen, 2009). Environmental stressors known to elevate GC concentrations include conditions when competition for food is high or food abundance is low or unpredictable (Chinnadurai et al., 2009; Goymann et al., 2001; Jeanniard du Dot et al., 2009; Kitaysky et al., 2010), when predation pressure is high (Bao et al., 2017; Creel et al., 2009; Sheriff et al., 2015), when social environments are challenging (Creel et al., 2013; Hennessy et al., 2009; Hofer and East, 2012), unstable or unpredictable (Bassett and Buchanan-Smith, 2007; Benhaiem et al., 2012; 2013; Goymann et al., 2001; Tung et al., 2012) and when males compete for receptive females (Mooring et al., 2006; Pavitt et al., 2015).

In vertebrates the energetic costs of different life history stages vary and transitions between life history stages are often marked by glucocorticoid mediated metabolic changes (Crespi et al., 2013; McEwen and Wingfield, 2003). In mammals, lactation is the most energetically costly component of reproduction for females (Martin, 1984). In large herbivores that produce relatively large precocial offspring, late gestation can also be an energetically costly life stage (Fisher et al., 2002).

In wildlife, measurement of circulating GC usually requires interventions (capture, restraint and blood sampling) which can elevate GC, thereby affecting GC measurements (Kersey and Dehnhard, 2014). Also, pulsatile, episodic or diurnal GC secretion may affect measures of serum glucocorticoid levels (Aurich et al., 2015; Kersey and Dehnhard, 2014; Schmidt et al., 2010). Quantification of fecal glucocorticoid metabolites ( $\mathrm{fGCM}$ ) dispenses with the need for interventions and provides a cumulative $\mathrm{fGCM}$ concentration produced during the time of gut passage (Ganswindt et al., 2012; Kersey and Dehnhard, 2014; Schwarzenberger, 2007). Although GC responses to stressors are commonly measured in terms of fGCM concentrations (Goymann, 2012; Graham and Brown, 1996), species specific differences in steroid metabolism requires that an enzyme immune assay (EIA) must be validated for the study species to which it is applied (Touma and Palme, 2005). This typically involves an experimental adrenocorticotropic hormone (ACTH) challenge conducted on individuals of the target species in captivity (Bashaw et al., 2016; Benhaiem et al., 2012).

Most plains zebra (Equus quagga) (hereafter termed zebra) in the Serengeti ecosystem, in East Africa ( 200,000 animals; IUCN, 2017) undertake a biennial migration in response to seasonal patterns of precipitation (Maddock, 1979; McNaughton, 1985). At the start of the wet season (in approximately November), zebra move from their dry season range in the north and west of the ecosystem through a transitional area to the short grass plains in the south-east of the ecosystem (Maddock, 1979), where they graze on vegetation containing essential minerals for pregnancy and lactation. As these 
minerals only occur in a few 'hotspots' elsewhere (McNaughton, 1990) they must be acquired in specific areas even though energy requirements can be fulfilled in many areas (MacNaughton and Banyikwa, 1995). When the wet season ends in May or early June, zebras leave the arid south-east and return to the north and west (Norton-Griffiths et al., 1975) which receives a higher annual rainfall than the south-east, and contains sources of surface water in the dry season (Kingdon, 1979). These migratory movements result in substantial local variation in the abundance and dispersion of zebra (Maddock, 1979; McNaughton, 1985). The dominant grass species and vegetation community on the short grass plains differs from those in the long grassland and woodland communities (MacNaughton and Banyikwa, 1995).

Plains zebras form stable groups termed family bands that contain one stallion and one to several unrelated mares and their subadult offspring. Stable social relationships and strong cohesion between females provides the core structure to zebra family bands and the socially dominant female in a band typically determines the direction of band movement (Klingel, 1969a, 1969b). Agonistic interactions can occur between the female members of one family band and those in another band (Klingel, 1969b). Band stallions rarely actively defend mature female band members whereas both stallions and bachelors do compete to recruit adolescent mares in estrus (Klingel, 1969b). Females use body posture and olfactory cues to advertise when they are in estrus (Klingel, 1969b). Males leave their natal band when between one and four years old (hereafter termed bachelor males) and join bachelor groups that have stable membership and stable within group social relationships (Klingel, 1969b; Rubenstein, 1986). The stability of bachelor groups is unique among equids (Linklater, 2000; Ransom and Kaczensky, 2016). It is thought that bachelor males cannot successfully compete for access to receptive females before they are about 4 years old (Klingel, 2013, 2010). Females usually leave their natal family band during their first estrus when approximately 13 months old and few conceive before they are over two years old (Klingel, 1969a). In the Serengeti ecosystem there is a marked birth peak in the wet season which typically occurs on the short grass plains, although foals are born throughout the year. Pregnancy lasts 360-396 days, and lactation 7-11 months (Wackernagel, 1965), with postpartum estrus occurring approximately one week after birth (Klingel, 1969a).

In several mammalian species, GC concentrations are elevated when population densities are high or are experimentally increased (Boonstra and Boag, 1992; Caslini et al., 2016; Forristal et al., 2012; He et al., 2014; Rogovin et al., 2003). Agonistic interactions are typically elevated when competition for resources, such as food or mates is increased at higher population densities. Furthermore, for animals living in stable groups, the coalescence of groups into large aggregations will lead to a decline in the predictability of the social environment. Disentangling the effects of these environmental stressors on GC concentrations is problematic. In the Serengeti ecosystem, the migratory movements of zebra cause large fluctuations in the number of zebra in different locations (Maddock, 1984; McNaughton, 1985) but little is known how aggregation affects GC concentrations in migratory zebras.

This study assesses the impact of life history stage and environmental stressors on the allostatic load of migratory zebra. Energetically costly life history stages should increase allostatic load hence we expect higher fGCM levels in females during late pregnancy and lactation than in mature females in other life stages. As stallions aim to prevent estrus females in their band from mating with rival males (either bachelor males or other band stallions) and they also compete for estrus females in other family bands, we predict higher fGCM concentrations in family band stallions than in bachelors. We 
also consider the impact of three environmental stressors (feeding competition, predation pressure and an unpredictable social environment) on fGCM concentrations. We expect the highest fGCM response to these three stressors to occur in the largest category of zebra aggregations. More specifically we expect: 1) feeding competition to increase with aggregation size in adult animals; 2 ) predation pressure to increase when numerous predators, particularly 'commuting' spotted hyenas (Crocuta crocuta) are attracted to large aggregations of prey (Hofer and East, 1995, 1993). More specifically we expected predation pressure to be most pronounced in large aggregations in all age and sex categories, and for predation pressure to be similar in medium and small aggregations; 3 ) the negative effect of unpredictable social relationships to increase as the size of zebra aggregations increase. Strong social bonds between mature band females, mothers and their offspring, and males within bachelor groups should to some extent buffer zebra against this social stressor, at least in small and medium aggregations.

\section{Materials and Methods}

\section{Study site and sample collection}

This study was conducted in the Serengeti NP, Tanzania, during the months of January-March, MayJuly and October 2016. In 2016, an El Niño climatic event resulted in above average rainfall in the Serengeti NP and an extension of rain into the dry season.

Zebras were sampled in two distinct habitat categories: the short grass plains in the south, and long grass, open woodland savannah in the center of the park (henceforth termed woodland boundary). Fecal samples were collected opportunistically, together with information on age, sex and reproductive state for sampled individuals, habitat type (short grass plains or woodland boundary) and the aggregation of zebras in the area. Zebra abundance within approximately $500 \mathrm{~m}$ in all directions of the research vehicle was estimated and categorized within three easily determined aggregation categories: small ( $\leq 50$ zebras), medium ( $>50$ and $\leq 200$ zebras), and large ( $>200$ zebras). In total 270 fecal samples were collected (in large $\mathrm{N}=124$, medium $\mathrm{N}=67$, small $\mathrm{N}=79$ aggregations of zebras). Following Klingel (1969b) we scored the age of animals in three categories: adult, subadult and foal using withers height and pelage (see fig. 1). Reproductive state in adult females was visually assessed in three categories (fig. 1): (i) lactating - mares accompanied by a suckling foal; (ii) late pregnant - mares with a conspicuously enlarge abdomen, indicating the presence of a large fetus and presumably $>6^{\text {th }}$ month of pregnancy; (iii) other - mares in neither of these two states, including earlier stages of pregnancy, and mares in estrus. Adult males were categorized as family stallions or bachelors (fig. 1). Definitions of each respective age class and reproductive state are shown in table 1.

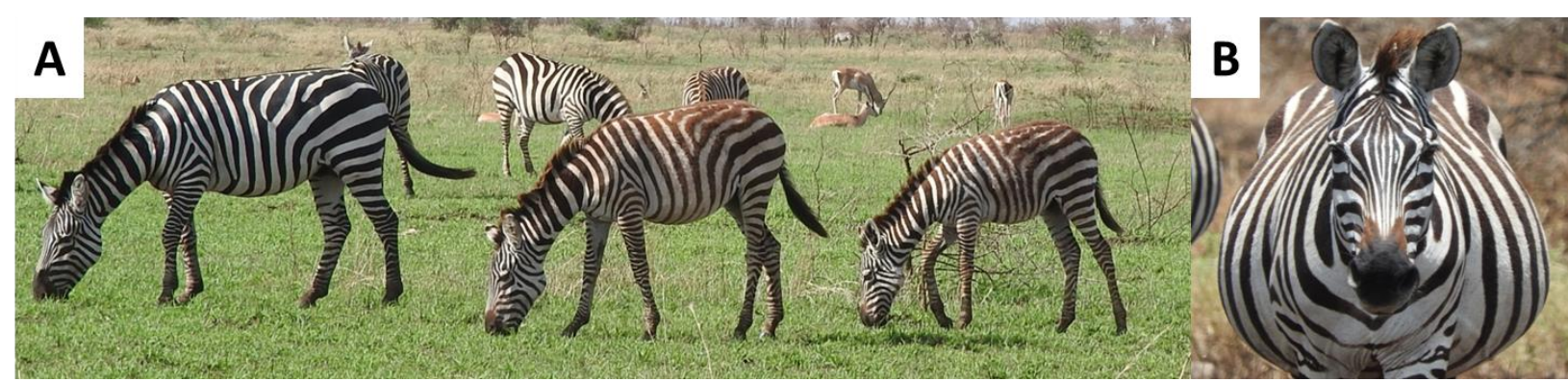

Figure 1 
Age and reproductive categories in plains zebra. (A) Left to right: mature female, subadult, foal, as assessed by withers height. (B) Late-pregnant mare, with enlarged abdomen.

Fecal samples were collected opportunistically immediately after deposition from individuals within approximately $150 \mathrm{~m}$. Care was taken to avoid soil contamination. To control for unequal distribution of fGCM in a fecal bolus, material was collected from several remote parts of each bolus then pooled and thoroughly mixed. The sample was immediately stored on ice in the field up to 8 hours and subsequently frozen for storage and shipment on dry ice until extraction at the Leibniz Institute for Zoo and Wildlife Research, Berlin (IZW). One sample was excluded from the final analysis due to a uterine prolapse in the respective mare after recently giving birth, as injuries and subsequent inflammation have been shown to lead to elevated fGCM levels in wildlife (Ganswindt et al., 2010).

\section{Table 1}

Categories of plains zebra by age class, sex, and reproductive state. Foals and subadults were combined in the age category termed juveniles.

\begin{tabular}{|c|c|c|c|}
\hline Age & Sex & Reproductive state & Definition \\
\hline foal & \multirow[b]{2}{*}{$\begin{array}{l}\text { not } \\
\text { determined }\end{array}$} & \multirow[b]{2}{*}{$\begin{array}{l}\text { not } \\
\text { applicable }\end{array}$} & withers height less than $80 \%$ of a mature individual \\
\hline subadult & & & $\begin{array}{l}\text { not smaller than } 80 \% \text { of the withers height of an adult but not } \\
\text { fully grown regarding overall body mass; residual patches of long } \\
\text { brownish 'foal fur' on the back }\end{array}$ \\
\hline \multirow{5}{*}{ mature } & \multirow{2}{*}{ Male } & bachelor & in a group with other males (adults and/or subadults) \\
\hline & & family stallion & in a group with 1 or more mares (and offspring) \\
\hline & \multirow{3}{*}{ Female } & late pregnant & $\begin{array}{l}\text { distended abdomen larger than well-nourished individuals, typical } \\
\text { 'pear shape' }\end{array}$ \\
\hline & & lactating & mare with foal \\
\hline & & other & no observable evidence of pregnancy and no associated foal \\
\hline
\end{tabular}

\section{Aggression rates between adult males}

To assess the rate of overt male-male agonistic interactions, males (bachelors and stallions) were observed from the research vehicle during a total observation period of $123 \mathrm{~h} 43 \mathrm{~min}$ (66h $51 \mathrm{~min}$ focused on stallions; $56 \mathrm{~h} 52 \mathrm{~min}$ focused on bachelor males). Low level approach-retreat interactions (displacements) lacking aggressive behaviors were not recorded. The animals being scanned for aggressive behaviors were changed at 10 min intervals. Play chases and play fights among bachelor males within a group and play fights initiated by a subadult male with the stallion within a family group were not recorded. We only recorded incidences of aggression that involved higher speed chases and aggressive behaviors such as kicking and biting.

\section{EIA validation and fGCM extraction}

We validated an enzyme-immunoassay (EIA) based on an antibody against 11ßhydroxyetiocholanolone (Pribbenow et al., 2014) for zebra. We obtained fecal samples from an ACTH challenge experiment conducted in 2011 on two captive zebras (one mare, one stallion) at the National Zoological Gardens of South Africa, Pretoria (Fourie, 2012; Périquet et al., 2017). The animals were immobilized and moved to the zoo's veterinary hospital for the experiment, and were housed there in separated, adjoining enclosures. After 9 and 10 days, respectively, each animal was again anesthetized and received an intramuscular injection of a synthetic correlate to 
adrenocorticotropic hormone (1 IU/kg SYNACTHEN DEPOT; tetracosactide, Novartis, South Africa). Fecal samples were collected from 30 hours before, to two days after SYNACTHEN administration. Fecal glucocorticoid metabolites were extracted as described in Périquet et al. (2017) and stored at $20{ }^{\circ} \mathrm{C}$ (Ganswindt et al., 2002) until shipment to the IZW. Respective fGCM concentrations of the ACTH challenge experiment are reported as $\mathrm{ng} / \mathrm{g}$ of dry weight (dw). We calculated baseline fGCM levels by using an iterative process excluding all peaks which were defined as greater than the mean+1.5 SD (de Bruin et al., 2014).

From wild zebras, sample aliquots of $0.5 \mathrm{~g}$ wet weight $(\mathrm{ww})$ were extracted with $4.5 \mathrm{ml}$ methanol (90\%) by shaking for $30 \mathrm{~min}$. Samples were centrifuged at $3000 \mathrm{rpm}$ for $15 \mathrm{~min}$, and $0.5 \mathrm{ml}$ of the supernatant diluted 1:1 with water for the subsequent EIA. Resulting fGCM concentrations are reported as ng/g ww.

\section{Statistical analysis}

To examine the contribution of potential environmental (including social) stressors and life history stages to fGCM concentrations in zebras in the Serengeti ecosystem, we fitted general linear models with log-transformed fGCM concentrations as the response variable. We fitted separate models for adult mares, adult males and juvenile animals (i.e. foals and subadults), respectively. We included in our models the following predictors: to test for the effects of aggregation we included a categorical measure of aggregation size (large, medium and small); to examine for effect of life history stages we included the two reproductive states for adult males (bachelor males and stallions associated with band females) and three reproductive states for adult females (lactation, late pregnancy and other) and two age classes (foal and subadult) for juvenile animals; to test for potential habitat effects, which might affect feeding competition, we included the two main habitat types (short grass plains and woodland boundary).

To assess the statistical significance of the predictor variables we used F-tests to compare the full model to reduced models in which the respective predictor was removed. Significant differences between predictor variables were analyzed post-hoc by a Tukey's Multiple Comparison of Means test. Visual inspections of all models indicated no obvious violations of assumptions of normality and homogeneity of error variances. Inspection of variance inflation factors (all below 1.80) did not indicate any problem of multicollinearity in any of the models. As zebras constantly roam and sites where feces were collected were frequently changed, the probability of repeated sampling of any one individual was considered negligible. Statistical analyses were performed in $R$ version 3.2.5 ( $R$ Development Core Team, 2016). The variance inflation factor was calculated using the $R$ package 'car' version 2.1-4 (Fox and Weisberg, 2011). The significance threshold was set at an $\alpha$-level of $p<0.05$. To quantify effect sizes of the predictors we calculated partial eta-squared $\left(\eta^{2}\right)$, which quantifies the proportion of variation explained by this predictor, while partialling out the effects of the other predictors (Muller \& Peterson, 1984). To quantify effect sizes in pairwise post-hoc tests, we calculated Cohen's d, which quantifies the difference in means normalized by the pooled standard deviation (Cohen, 1988). These measures were computed using the heplots v1.3-4 (Fox et al., 2009), and Isr v0.5 packages (Navarro, 2015). 


\section{Results}

\section{ACTH challenge and EIA validation}

For the animals subjected to the ACTH challenge experiment, the respective $\mathrm{fGCM}$ baseline concentrations were $1,403 \mathrm{ng} / \mathrm{g} \mathrm{dm}$ (male), and $844 \mathrm{ng} / \mathrm{g} \mathrm{dm}$ (female). The 11ßhydroxyetiocholanolone EIA showed fGCM peaks at $27,42,47$, and 48 hours post-injection in the stallion, and after 29 hours in the mare (fig. 2). A return to baseline levels could not be determined in the stallion, but was found in the mare after 42 hours. Maximum peak levels of 2,440 ng/g (42 hours post-injection) and 1,385 ng/g corresponding to a $183 \%$ and $164 \%$ increase above baseline were reached in the male and the female, respectively. The ACTH stimulated increase in fGCM levels suggests that the fecal metabolites traced by the assay are adequate indicators of adrenal activity in zebras. Thus the $11 ß$-hydroxyetiocholanolone EIA was used for further analyses. Mean inter-assay variance was $14.3 \%$, based on four control samples.

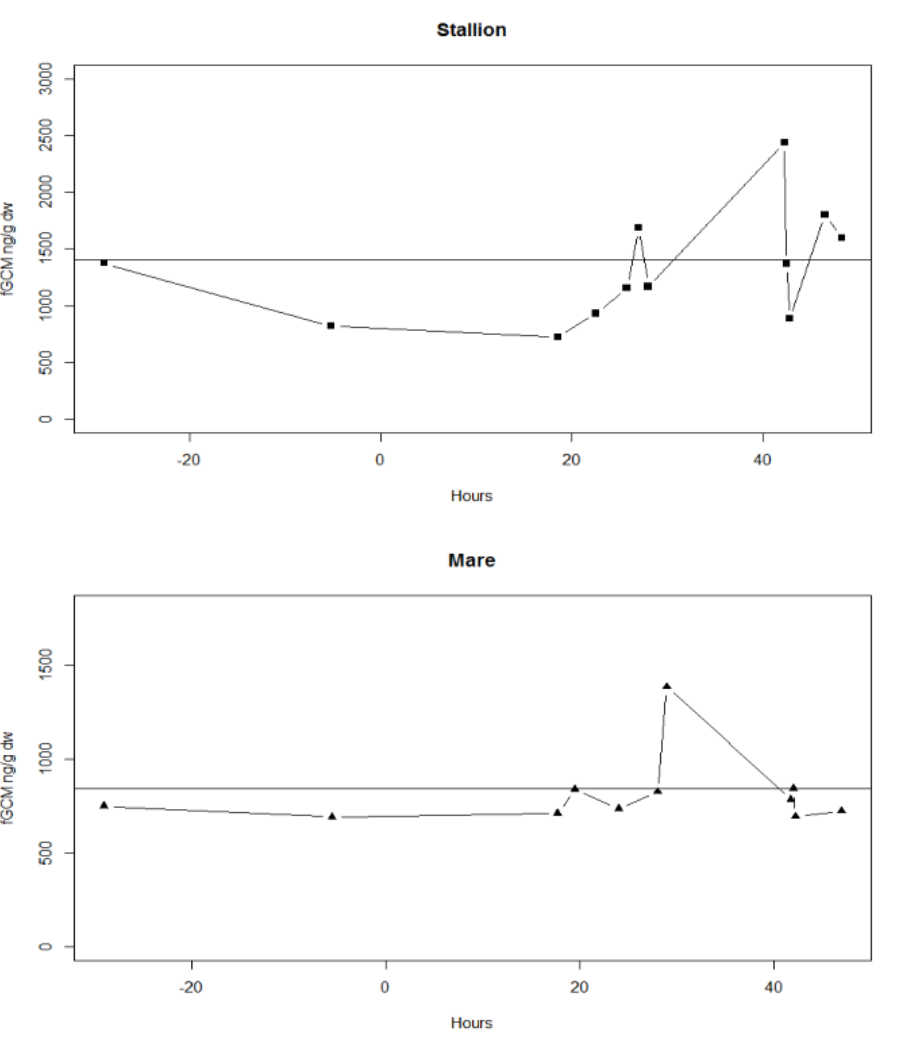

\section{Figure 2}

ACTH challenge experiment on captive plains zebras. Course of fecal glucocorticoid metabolite ( $\mathrm{fGCM}$ ) concentrations ( $\mathrm{ng} / \mathrm{g}$ dry weight) in fecal samples collected during a period spanning 29 hours before to 48 hours after the administration of ACTH (hour 0) applying an enzyme immunoassay based on an antibody against $11 \beta$-hydroxyetiocholanolon in a zebra stallion and a zebra mare, respectively. The horizontal line indicates the calculated individual baseline $\mathrm{fGCM}$ concentrations.

fGCM levels in wild adult plains zebra males

In adult males $(\mathrm{N}=87)$, the size category of aggregation was a significant predictor of $\mathrm{fGCM}$ levels ( $p=0.007$; table 2, fig. 3). Post-hoc tests revealed significantly higher fGCM concentrations in males in large aggregations than in medium aggregations $(p=0.032$, table 3$)$, and in large than small 
aggregations $(p=0.012)$. The difference in $\mathrm{fGCM}$ concentrations between males in medium and small aggregations was not significant. Life history stage was significantly positively correlated with fGCM concentrations in adult males ( $p=0.018$ ) with significantly higher fGCM levels in band stallions than in bachelors (fig. 4). The size of the effect of reproductive state in males was less $\left(\eta^{2}=0.08\right)$ than that of aggregation $\left(\eta^{2}=0.12\right.$, table 2$)$. Habitat type had no significant effect on fGCM concentrations $(p=0.143$, table 2$)$. The aggressive interaction rate among males was 0.04 per hour (5 interactions/123 hours $43 \mathrm{~min}$ ). All interactions were initiated by band stallions, and targets included one band stallion, and four bachelor males.

fGCM levels in wild adult plains zebra females

In adult females ( $\mathrm{N}=136)$ the size category of aggregation significantly affected $\mathrm{fGCM}$ levels $(p<0.001$; table 2). The post-hoc tests revealed significantly higher fGCM levels in large than in medium aggregations ( $p<0.001$, table 3$)$, and in large than in small aggregation $(p<0.001$, fig. 3$)$, whereas the difference between medium and small aggregations was not significant. In adult females, we did not detect a statistically significant effect of life history stage (lactating, in late pregnancy, and mares in neither of these two states) ( $p=0.759$, table 2$)$. No statistically significant differences were detected between habitat types ( $p=0.083$, table 2$)$.

fGCM levels in wild juvenile plains zebras

In juvenile zebras ( $\mathrm{N}=46$ ) fGCM concentrations were significantly affected by the size category of aggregation ( $p=0.009$; table 2 ), being significantly higher in large than in medium aggregations ( $p=0.009$; table 3 , fig. 3 ) whereas differences between large and small, and medium and small aggregations were not significant. Concentrations of fGCM were not significantly affected by categories of age $(p=0.952$, table 2$)$ or habitat type $(p=0.060$, table 2$)$.

\section{Table 2}

Results of general linear models that assess the effects of aggregation, reproductive state, and habitat type on fecal glucocorticoid metabolite concentrations (fGCM) in adult male, adult female and juvenile zebras (subadults and foals), respectively, and values for degrees of freedom (df), sample size $(N)$, and partial eta-squared (n2). Significant effects in bold.

\begin{tabular}{clcccc}
\hline \multirow{2}{*}{ Category } & Independent variable & df & $\mathbf{F}$ & $\begin{array}{c}\text { partial } \\
\mathbf{\eta}^{2}\end{array}$ & $\boldsymbol{p}$ \\
\hline \hline \multirow{3}{*}{$\begin{array}{c}\text { Adult males } \\
N=87\end{array}$} & Aggregation & 2 & $\mathbf{5 . 1 9}$ & $\mathbf{0 . 1 2}$ & $\mathbf{0 . 0 0 7}$ \\
\cline { 2 - 6 } & Reproductive state & 1 & $\mathbf{5 . 8 2}$ & $\mathbf{0 . 0 8}$ & $\mathbf{0 . 0 1 8}$ \\
\cline { 2 - 6 } & Habitat & 1 & 2.19 & 0.03 & 0.143 \\
\hline \multirow{2}{*}{$\begin{array}{c}\text { Adult females } \\
N=136\end{array}$} & Reproductive state & 2 & 0.28 & 0.004 & 0.759 \\
\cline { 2 - 6 } & Habitat & 1 & 3.06 & 0.02 & 0.083 \\
\hline Juvenile & Aggregation & 2 & $\mathbf{5 . 2 2}$ & $\mathbf{0 . 2 0}$ & $\mathbf{0 . 0 0 9}$ \\
\cline { 2 - 6 }$N=46$ & Age class & 1 & 0.004 & $<0.001$ & 0.952 \\
\cline { 2 - 6 } & Habitat & 1 & 4.07 & 0.09 & 0.060 \\
\hline
\end{tabular}

Table 3 
Results of post-hoc tests (Tukey's Multiple Comparison of Means) that were used to assess the effects of aggregation size on fGCM levels. Significant effects in bold. Values for Cohen's $d$ are also provided.

\begin{tabular}{clccccc}
\hline post-hoc tests & & & & & \\
\hline & Aggregation & Estimate & $\begin{array}{c}\text { Std. } \\
\text { Error }\end{array}$ & $\boldsymbol{t}$ & $\begin{array}{c}\text { Cohen's } \\
\boldsymbol{d}\end{array}$ & $\boldsymbol{p}$ \\
\hline \multirow{3}{*}{ Adult males } & small $\rightarrow$ large & $\mathbf{0 . 2 9 4}$ & 0.11 & 2.93 & $\mathbf{1 . 2 3}$ & $\mathbf{0 . 0 1 2}$ \\
& medium $\rightarrow$ large & $\mathbf{0 . 3 0 9}$ & 0.11 & 2.93 & $\mathbf{0 . 9 7}$ & $\mathbf{0 . 0 3 2}$ \\
& small $\rightarrow$ medium & 0.015 & 0.11 & 0.13 & 0.01 & 0.990 \\
\hline \multirow{3}{*}{ Adult females } & small $\rightarrow$ large & $\mathbf{0 . 3 6 3}$ & 0.07 & 5.36 & $\mathbf{1 . 3 2}$ & $<\mathbf{0 . 0 0 1}$ \\
& medium $\rightarrow$ large & $\mathbf{0 . 3 6 7}$ & 0.07 & 5.06 & $\mathbf{1 . 1 1}$ & $<0.001$ \\
& small $\rightarrow$ medium & 0.004 & 0.08 & 0.05 & 0.12 & 0.999 \\
\hline \multirow{2}{*}{ Juvenile } & small $\rightarrow$ large & 0.270 & 0.12 & 1.94 & 1.09 & 0.138 \\
& medium $\rightarrow$ large & $\mathbf{0 . 3 7 8}$ & 0.14 & 3.10 & $\mathbf{1 . 1 5}$ & $\mathbf{0 . 0 0 9}$ \\
& small $\rightarrow$ medium & 0.107 & 0.15 & 0.72 & 0.09 & 0.751 \\
\hline
\end{tabular}
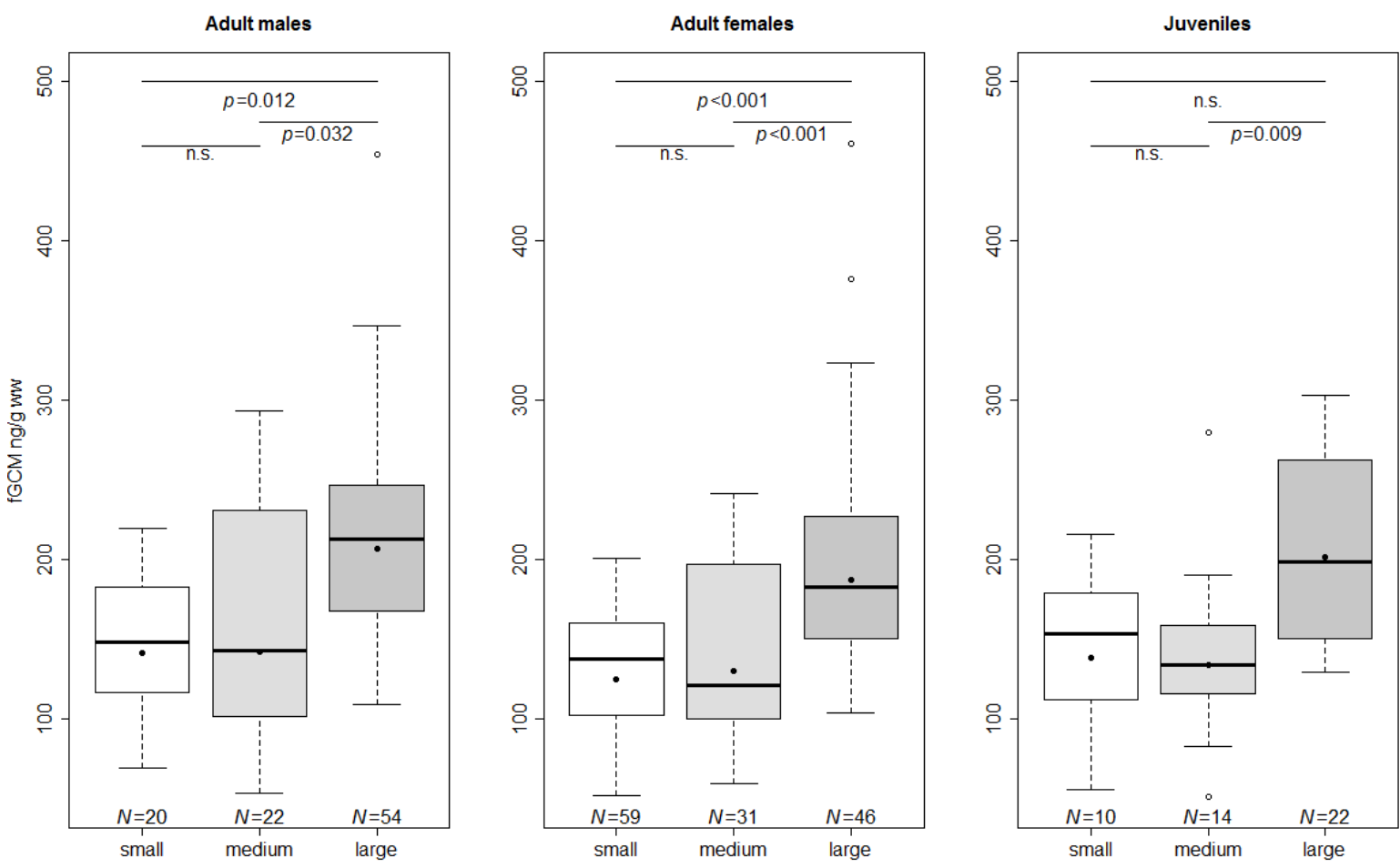

\section{Figure 3}

The effect of aggregation category on fGCM concentrations (ng/g ww) in adult male, adult female and juvenile plains zebras. Boxes indicate $2^{\text {nd }}$ and $3^{\text {rd }}$ quartiles, center lines indicate median values, upper (and lower) whiskers extend to the highest (and lowest) value that is within 1.5 times the inter-quartile range. Data points beyond the end of the whiskers are plotted as open dots. Filled dots indicate the predicted mean effects of aggregation by the respective linear model. 


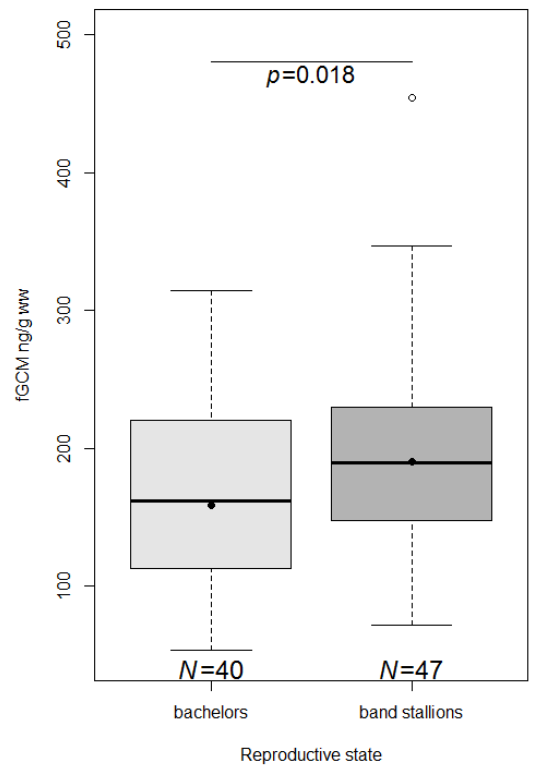

\section{Figure 4}

Effect of reproductive state on $\mathrm{fGCM}$ concentrations ( $\mathrm{ng} / \mathrm{g} \mathrm{ww}$ ) in adult male plains zebras (bachelors and stallions). Boxes indicate $2^{\text {nd }}$ and $3^{\text {rd }}$ quartiles, center lines indicate median values. Upper (and lower) whiskers extend to the highest (and lowest) value that is within 1.5 times the inter-quartile range. Data points beyond the end of the whiskers are plotted as open dots. Filled dots indicate the mean predicted effect of reproductive state by the linear model. 


\section{Discussion}

In this study we measured fGCM concentrations in zebra to assess the contribution of life history and environmental stressors to allostatic load in the migratory population in the Serengeti ecosystem. The results of an ACTH challenge experiment on two captive zebras show that an $11 \beta$ hydroxyetiocholanolone EIA is appropriate for the measurement of fGCM concentrations in this species. Following ACTH administration, $\mathrm{FGCM}$ concentrations increased above baseline concentrations by $183 \%$ and $164 \%$ in the male and the female, respectively. Results were presented per gram dry matter and assuming a moisture content in feces of about $50 \%$ (Ndlela and Schmidt, 2016), fGCM concentrations in the captive animals before the ACTH challenge (fig. 2) were considerably above those obtained from zebra in the Serengeti ecosystem (fig. 3) when adjusted for wet weight. This might be related to both captive zebras being moved from their social group and housed individually in adjoined enclosures for the time of the experiment, which in domestic horses can significantly increase fGCM concentrations (Yarnell et al., 2015).

Our findings revealed that fGCM concentrations were significantly elevated in zebra in the Serengeti ecosystem when they gathered in large aggregations (> 200 zebra), and this response was apparent in all age and sex categories and life history stages (table 2, fig. 3). Aggregation had a larger effect than any other predictor on allostatic load in adult male, adult female and juvenile zebra (table 2). This result was expected, as all three environmental stressors (feeding competition, predator pressure, and an unpredictable social environment) should have their strongest impact when zebras occur in large aggregations. Our results most likely represent the GC response of individuals to more than one stressor but it is difficult to disentangle the contribution of potential stressors to $\mathrm{fGCM}$ concentrations measured in free-ranging wildlife (Creel et al., 2013). Even so we use differences in the predicted and observed patterns of $\mathrm{FGCM}$ concentration in our age and sex categories to tentatively interpret our findings.

We found that $\mathrm{fGCM}$ concentrations of stallions associated with bands of females were significantly higher than those of bachelor males (fig. 4). This result is consistent with our prediction that the allostatic load of stallions should have a larger component induced by the social environment than that of bachelor males. Band stallions have to maintain their long-term social bonds with the female band members and challenge bachelor males that attempt to approach them (Fischhoff et al., 2009; Klingel, 1974, 1969b), as our limited results on male-male aggressive interactions show. Band stallions can also be challenged by stallions from other family bands (Klingel, 1969b). In contrast, bachelors do not maintain relationships with females, and have more opportunity to decide which zebras they interact with, challenge and avoid. Although behavioral differences between stallions and bachelors provide a plausible interpretation of our findings, more research is required to establish whether the high allostatic load of band stallions is primarily due to challenges by other male competitors (Goymann and Wingfield, 2004), rather than other activities, such as the defense of band members against attacks by predators (Kruuk, 1972). In domestic horses, reproductive function can be modulated by the social environment, and a change from non-breeding to breeding status coincides with a significant increase in androgen concentrations (McDonnell and Murray, 1995). However, in free-ranging equids, including zebras, bachelors are not necessarily reproductively inactive, as they do compete for access to receptive females. In fission-fusion groups of giraffe (Giraffa camelopardalis), male dominance is based on age and size, hence older larger males are socially dominant over younger smaller ones. In single sex groups, older dominant males have lower fGCM levels than younger ones, whereas in mixed sex group the reverse is true (Wolf et 
al., 2018), suggesting that when females are present, dominant males are more challenged by the presence of other males than when females are absent.

Lactation is an energetically demanding life history stage (Costa et al., 1986; Houston et al., 2007; Landete-Castillejos et al., 2002) and lactating females may resort to resource allocation trade-offs when their intake of food is insufficient to sustain lactation (Archie et al., 2014; East et al., 2015; Festa-Bianchet, 1989; Hamel and Côté, 2009). In species, such as zebra, that produce precocial offspring, the total mass of the contents of the uterus carried by a pregnant near-term female is roughly equivalent to $15 \%$ of the mother's body mass (Smuts, 1976), and this may also increase maternal allostatic load. Despite this, we found no evidence for fGCM concentrations being elevated in late-pregnant and lactating females (Table 2). This agrees with findings in domestic horses, where fGCM levels of mares did not differ between reproductive state, presumably because lactating females increased their food intake and decreased their time spent alert and socializing (York and Schulte, 2014). Endocrine adjustments which up-regulate the efficiency of protein, glucose and lipid metabolism, and decreases plasma leptin concentrations thereby promoting feed intake during the peripartum period may also help prevent energy deficits during lactation in horses (Arfuso et al., 2016; Heidler et al., 2003). Comparable behavioral and metabolic adjustments might also occur in zebras, but for these to prevent energy deficits, female zebras should have access to sufficient forage to permit an increase in food intake before resorting to the mobilization of energy from fat reserves. Our study period coincided with El Niño climatic conditions in the Serengeti ecosystem, hence there was an abundance of forage and zebras probably also had larger than normal fat reserves. In more typical years (i.e. longer dry seasons, and reduced growth of forage), fat reserves of females are probably more limited and females at energetically costly life history stages may experience periods when their energy intake is insufficient and this may be reflected in elevated fGCM concentrations.

Theoretically, animals that join a group can benefit from the 'dilution effect' and the 'many eyes effect'(Elgar, 1989; Rubenstein, 1978) . The dilution effect suggests that an individual's risk of predation should decrease with increasing group size and hence that its fGCM concentration should be lowest in large aggregations and highest in small aggregations. Similarly the 'many eyes effect' also suggests that fGCM concentration should be lowest in large aggregations because of the reduced vigilance and increase foraging. Our results do not support these expectations. Alternatively, when predators can detect prey more easily and their foraging efficiency is higher in large aggregations of prey than in smaller groupings (Taylor, 1979), then predators are drawn to areas containing large aggregations of prey. This idea predicts higher predation pressure in areas containing large aggregations of zebra than those with either medium or small aggregations. Lions (Panthera leo) and spotted hyenas are the main predators of zebra in the Serengeti ecosystem (Grange et al., 2004). Lions predominately hunt within the territory held by their pride, hence the predation pressure exerted by a pride of lions is mostly limited to its territory whereas nomadic male lions hunt over far larger areas (Schaller, 1972). The spotted hyena is the most numerous large carnivore in the Serengeti ecosystem (Hofer and East, 1995), and in the Serengeti NP this species has a unique long distance foraging behavior (termed commuting). Even though spotted hyena clans hold territories, individual clan members regularly conduct short-term, long distance (approximately 100 $\mathrm{km}$ round trip) foraging excursions to areas holding high densities of migratory herbivores (Hofer and East, 1993). Spotted hyenas are coursers that hunt solitarily or in small groups (East and Hofer, 2013; Kruuk, 1972), and clans are typically large containing approximately 60 adult animals (East et al., 2003). Although both, lions and spotted hyenas contributed to predator pressure on zebra during our study (Clinchy et al., 2013) we suggest that the large number of spotted hyenas, including both 
member of local clans and intruding commuters (Hofer and East, 1993) probably exerted the main predator pressure on zebras in large aggregations, thereby contributing to the elevated allostatic load of zebras in large aggregations.

The linear hierarchy amongst adult female members of a band (Klingel, 1972, 1969b) provides a predictable social environment for females and their offspring as do established relationships between males within stable bachelor groups. However, social relationships between females of different bands are not defined (Klingel, 1969b) and hence are less predictable, as are those of bachelor males to non-group members. As the size category of zebra aggregations increases, the predictability of the social environment is expected to decline which could elevate the GC response to this potential stressor. Our results do not fully support this idea as they did not reveal a significant increase in fGCM concentrations between small and medium aggregations in adult males, adult females or juveniles.

Our results indicate that in adult males both reproductive status and aggregation per se have a significant effect on fGCM concentrations, and that the effect of aggregation appears to be larger than that of reproductive status (table 2). This suggests that competitive male-male interactions do not entirely explain the effect of aggregation on fGCM concentrations in adult males (table 2) and that other stressors, such as predator pressure and possibly feeding competition also contribute. Even so, there is evidence from other species that aggregation can be stressful. For example, when local densities of red deer (Cervus elaphus) are increased by supplementary feeding, fGCM concentrations are $31-43 \%$ higher than in control areas and this response is not significantly affected by aggression rates at feeding sites (Forristal et al., 2012). Furthermore, social instability caused by changes in group composition can act as a stressor in feral and domestic horses (e.g. Alexander and Irvine, 1998; Nuñez et al., 2014; York and Schulte, 2014) hence the unpredictable social environment in large zebra aggregations may contribute to the elevated fGCM concentrations we report for large aggregations.

In the wet season, zebras (and other migratory herbivores) migrate south and aggregate on the short grass plains to feed on highly nutritious forage containing essential minerals for pregnant and lactating females, and young animals (McNaughton, 1990). Our results reveal a non-significant trend for higher fGCM concentrations in both adult females and juveniles (but not adult males) when on the short grass plains than in the woodland. This possibly suggests a low degree of feeding competition for nutritious forage among adult females and juveniles, even during El Niño climatic conditions. This trend might be more pronounced when wet season precipitation is below average. In general, feeding competition in large aggregations might increase the energetic costs of foraging because zebras probably need to move more often to ingest sufficient food in heavily grazed areas. Furthermore, because food intake per bite is likely to be substantially lower when zebras crop short sward than long grass, zebras probably move need to more frequently when grazing on the short grass plains than in long grass areas.

Zebras are hindgut fermenters that can extract nutrients from low quality, fiber rich vegetation (Duncan et al., 1990), but in comparison to many ruminants, they shed more water in their feces (Cain et al., 2012; Woodall and Skinner, 1993). Under typical dry season conditions, fGCM concentrations would be expected to increase when large aggregations of zebra compete for access to declining surface water supplies and the lower nutrient forage available in a typical dry season (Fischhoff et al., 2009). However, the El Niño climatic conditions during the current study resulted in 
increased rainfall, including precipitation during several dry season months. Hence the typical adverse effect of the dry season on forage and surface water availability were considerably reduced and we found no evidence for the expected increase in $\mathrm{FGCM}$ concentrations in their dry season habitat. Studies on zebra in Namibia (Cizauskas et al., 2015) and South Africa (Chinnadurai et al., 2009) have reported a significant effect of drought-associated change in forage quality on fGCM levels in zebras. The El Niño conditions enabled us to measure aggregation associated stress independent of drought associated changes.

In conclusion, we found that the allostatic load of migratory zebras in large aggregations is significantly elevated. This probably represents the combined energetic cost of responses to several stressors, including foraging competition, predation pressure and unpredictable social environments. The contribution of these stressors to the allostatic load of zebra requires further investigation particularly during years when climatic conditions during the dry season are more typical, and during wet seasons when precipitation is below average. Significant difference in fGCM concentrations between life history stages in adult males and not female also requires further research to establish to what extent climatic conditions alter the ability of individuals to fulfill the cost of reproduction in this species.

\section{Acknowledgments}

We thank the Tanzania Commission of Science and Technology, Tanzania National Parks and the Tanzania Wildlife Research Institute for their support of this study. We are grateful to Dagmar Thierer, Stephan Karl and Katrin Paschmionka at the IZW for technical assistance and to Annie Francis, Thomas Shabani, Tom Morrison, Sonja Metzger, and Heribert Hofer for assistance in Tanzania. We thank Kim Wallen, and two anonymous reviewers for their constructive comments.

\section{Funding}

This study was supported by a grant from the Leibniz Gemeinschaft (SAW-2015-IZW-1 440) and the Leibniz Institute for Zoo and Wildlife Research.

\section{Ethical standards}

All protocols were non-invasive and adhered to the laws and guidelines of Tanzania. Permission to conduct research in Tanzania was granted to PAS and MLE by the Tanzania Commission for Science and Technology (permit No. 2015-168-NA-90-130). Permission to undertake research within the Serengeti National Park was granted to PAS and MLE by the Tanzanian National Parks Authority, and the research was approved by the Tanzanian Wildlife Research Institute. This study was approved by the Internal Committee for Ethics and Animal Welfare of the Leibniz-Institute for Zoo and Wildlife Research, approval number 2015-09-02. 


\section{References}

Alexander, S.L., Irvine, C.H.G., 1998. The effect of social stress on adrenal axis activity in horses: The importance of monitoring corticosteroid-binding globulin capacity. J. Endocrinol. 157, 425-432. doi:10.1677/joe.0.1570425

Archie, E.A., Altmann, J., Alberts, S.C., 2014. Costs of reproduction in a long-lived female primate: injury risk and wound healing. Behav. Ecol. Sociobiol. 68, 1183-1193. doi:10.1007/s00265-0141729-4

Arfuso, F., Giannetto, C., Rizzo, M., Fazio, F., Giudice, E., Piccione, G., 2016. Serum levels of mitochondrial uncoupling protein 1 , leptin, and lipids during late pregnancy and the early postpartum period in mares. Theriogenology 86, 1156-1164. doi:10.1016/j.theriogenology.2016.04.005

Aurich, J., Wulf, M., Ille, N., Erber, R., von Lewinski, M., Palme, R., Aurich, C., 2015. Effects of season, age, sex, and housing on salivary cortisol concentrations in horses. Domest. Anim. Endocrinol. 52, 11-16. doi:10.1016/j.domaniend.2015.01.003

Bao, H., Fryxell, J.M., Liu, H., Dou, H., Ma, Y., Jiang, G., 2017. Effects of interspecific interaction-linked habitat factors on moose resource selection and environmental stress. Sci. Rep. 7, 41514. doi:10.1038/srep41514

Bashaw, M.J., Sicks, F., Palme, R., Schwarzenberger, F., Tordiffe, A.S.W., Ganswindt, A., 2016. Noninvasive assessment of adrenocortical activity as a measure of stress in giraffe (Giraffa camelopardalis). BMC Vet. Res. 12, 235. doi:10.1186/s12917-016-0864-8

Benhaiem, S., Dehnhard, M., Bonanni, R., Hofer, H., Goymann, W., Eulenberger, K., East, M.L., 2012. Validation of an enzyme immunoassay for the measurement of faecal glucocorticoid metabolites in spotted hyenas (Crocuta crocuta). Gen. Comp. Endocrinol. 178, 265-271. doi:10.1016/j.ygcen.2012.05.006

Benhaiem, S., Hofer, H., Dehnhard, M., Helms, J., East, M.L., 2013. Sibling competition and hunger increase allostatic load in spotted hyaenas. Biol. Lett. 9, 20130040. doi:10.1098/rsbl.2013.0040

Boonstra, R., Boag, P.T., 1992. Spring declines in Microtus pennsy/vanicus and the Role of Steroid Hormones. J. Anim. Ecol. 61, 339-352. doi:10.2307/5326

Cain, J.W., Owen-Smith, N., Macandza, V.A., 2012. The costs of drinking: comparative water dependency of sable antelope and zebra. J. Zool. 286, 58-67. doi:10.1111/j.14697998.2011.00848.x

Caslini, C., Comin, A., Peric, T., Prandi, A., Pedrotti, L., Mattiello, S., 2016. Use of hair cortisol analysis for comparing population status in wild red deer (Cervus elaphus) living in areas with different characteristics. Eur. J. Wildl. Res. 62, 713-723. doi:10.1007/s10344-016-1049-2

Chinnadurai, S.K., Millspaugh, J.J., Matthews, W.S., Canter, K., Slotow, R., Washburn, B.E., Woods, R.J., Article, T., 2009. Validation of fecal glucocorticoid metabolite assays for South African herbivores. J. Wildl. Manage. 73, 1014-1020. doi:10.2193/2008-430

Cizauskas, C. a., Turner, W.C., Pitts, N., Getz, W.M., 2015. Seasonal patterns of hormones, macroparasites, and microparasites in wild African ungulates: the interplay among stress, reproduction, and disease. PLoS One 10, e0120800. doi:10.1371/journal.pone.0120800

Clinchy, M., Sheriff, M.J., Zanette, L.Y., 2013. Predator-induced stress and the ecology of fear. Funct. Ecol. 27, 56-65. doi:10.1111/1365-2435.12007

Costa, D.P., Boeuf, B.J. Le, Huntley, A.C., Ortiz, C.L., 1986. The energetics of lactation in the Northern 
elephant seal, Mirounga angustirostris. J. Zool. 209, 21-33. doi:10.1111/j.1469-

7998.1986.tb03563.x

Creel, S., Dantzer, B., Goymann, W., Rubenstein, D.R., 2013. The ecology of stress: effects of the social environment. Funct. Ecol. 27, 66-80. doi:10.1111/j.1365-2435.2012.02029.x

Creel, S., Winnie, J.A., Christianson, D., 2009. Glucocorticoid stress hormones and the effect of predation risk on elk reproduction. Proc. Natl. Acad. Sci. 106, 12388-12393. doi:10.1073/pnas.0902235106

Crespi, E.J., Williams, T.D., Jessop, T.S., Delehanty, B., 2013. Life history and the ecology of stress: how do glucocorticoid hormones influence life-history variation in animals? Funct. Ecol. 27, 93106. doi:10.1111/1365-2435.12009

de Bruin, P.R., Ganswindt, A., Bennett, N.C., Medger, K., 2014. The pattern of ovulation in the southern African spiny mouse (Acomys spinosissimus). Mamm. Biol. - Zeitschrift für Säugetierkd. 79, 318-324. doi:10.1016/j.mambio.2014.05.003

Duncan, P., Foose, T.J., Gordon, I.J., Gakahu, C.G., Lloyd, M., 1990. Comparative nutrient extraction from forages by grazing bovids and equids: a test of the nutritional model of equid/bovid competition and coexistence. Oecologia 84, 411-418. doi:10.1007/BF00329768

East, M.L., Burke, T., Wilhelm, K., Greig, C., Hofer, H., 2003. Sexual conflicts in spotted hyenas: male and female mating tactics and their reproductive outcome with respect to age, social status and tenure. Proc. R. Soc. B Biol. Sci. 270, 1247-1254. doi:10.1098/rspb.2003.2363

East, M.L., Hofer, H., 2013. Crocuta crocuta Spotted Hyena, in: Kingdon, J., Hoffman, M. (Eds.), Mammals of Africa. Volume V: Carnivores, Pangolins, Equids and Rhinoceroses. Bloomsbury, London, pp. 273-281.

East, M.L., Otto, E., Helms, J., Thierer, D., Cable, J., Hofer, H., 2015. Does lactation lead to resource allocation trade-offs in the spotted hyaena? Behav. Ecol. Sociobiol. 69, 805-814. doi:10.1007/s00265-015-1897-x

Elgar, M., 1989. Predator vigilance and group size in mammals and birds: a critical review of the empirical evidence. Biol. Rev. 64, 13-33. doi:10.1111/j.1469-185X.1989.tb00636.x

Festa-Bianchet, M., 1989. Individual differences, parasites, and the costs of reproduction for bighorn ewes (Ovis canadensis). J. Anim. Ecol. 58, 785-795. doi:10.2307/5124

Fischhoff, I.R., Dushoff, J., Sundaresan, S.R., Cordingley, J.E., Rubenstein, D.I., 2009. Reproductive status influences group size and persistence of bonds in male plains zebra (Equus burchelli). Behav. Ecol. Sociobiol. 63, 1035-1043. doi:10.1007/s00265-009-0723-8

Fisher, D.O., Blomberg, S.P., Owens, I.P.F., 2002. Convergent maternal care strategies in ungulates and macropods. Evolution. 56, 167-176. doi:10.1554/00143820(2002)056[0167:CMCSIU]2.0.CO;2

Forristal, V.E., Creel, S., Taper, M.L., Scurlock, B.M., Cross, P.C., 2012. Effects of supplemental feeding and aggregation on fecal glucocorticoid metabolite concentrations in elk. J. Wildl. Manage. 76, 694-702. doi:10.1002/jwmg.312

Fourie, C.E., 2012. Vigilance behaviour and its endocrine correlates in Plains zebra (Equus burchelli) living in a predator-free landscape. MSc dissertation at the University of Pretoria.

Fox, J., Friendly, M., Monette, G., 2009. Visualizing hypothesis tests in multivariate linear models: the heplots package for R. Comput. Stat. 24, 233-246. doi:10.1007/s00180-008-0120-1 
Fox, J., Weisberg, S., 2011. An R companion to applied regression, 2nd editio. ed. Thousand Oaks, CA.

Ganswindt, A., Brown, J.L., Freeman, E.W., Kouba, A.J., Penfold, L.M., Santymire, R.M., Vick, M.M., Wielebnowski, N., Willis, E.L., Milnes, M.R., 2012. International Society for Wildlife Endocrinology: the future of endocrine measures for reproductive science, animal welfare and conservation biology. Biol. Lett. 8, 695-7. doi:10.1098/rsbl.2011.1181

Ganswindt, A., Heistermann, M., Borragan, S., Hodges, J.K., 2002. Assessment of testicular endocrine function in captive African elephants by measurement of urinary and fecal androgens. Zoo Biol. 21, 27-36. doi:10.1002/zoo.10034

Ganswindt, A., Münscher, S., Henley, M., Palme, R., Thompson, P., Bertschinger, H., 2010. Concentrations of faecal glucocorticoid metabolites in physically injured free-ranging African elephants Loxodonta africana. Wildlife Biol. 16, 323-332. doi:10.2981/09-081

Goymann, W., 2012. On the use of non-invasive hormone research in uncontrolled, natural environments: The problem with sex, diet, metabolic rate and the individual. Methods Ecol. Evol. 3, 757-765. doi:10.1111/j.2041-210X.2012.00203.x

Goymann, W., East, M.L., Wachter, B., Honer, O.P., Mostl, E., Van't Holf, T.J., Hofer, H., 2001. Social, state-dependent and environmental modulation of faecal corticosteroid levels in free-ranging female spotted hyenas. Proc. R. Soc. B Biol. Sci. 268, 2453-2459. doi:10.1098/rspb.2001.1828

Goymann, W., Wingfield, J.C., 2004. Allostatic load, social status and stress hormones: the costs of social status matter. Anim. Behav. 67, 591-602. doi:10.1016/j.anbehav.2003.08.007

Graham, L.H., Brown, J.L., 1996. Cortisol metabolism in the domestic cat and implications for noninvasive monitoring of adrenocortical function in endangered felids. Zoo Biol. 15, 71-82.

Grange, S., Duncan, P., Gaillard, J.-M., Sinclair, A.R.E., Gogan, P.J.P., Packer, C., Hofer, H., East, M., 2004. What limits the Serengeti zebra population? Oecologia 140, 523-532. doi:10.1007/s00442-004-1567-6

Hamel, S., Côté, S.D., 2009. Foraging decisions in a capital breeder: trade-offs between mass gain and lactation. Oecologia 161, 421-432. doi:10.1007/s00442-009-1377-y

He, L., Wang, W.X., Li, L.H., Liu, B.Q., Liu, G., Liu, S.Q., Qi, L., Hu, D.F., 2014. Effects of crowding and sex on fecal cortisol levels of captive forest musk deer. Biol. Res. 47, 4-9. doi:10.1186/0717$6287-47-48$

Heidler, B., Parvizi, N., Sauerwein, H., Bruckmaier, R.M., Heintges, U., Aurich, J.E., Aurich, C., 2003. Effects of lactation on metabolic and reproductive hormones in Lipizzaner mares. Domest. Anim. Endocrinol. 25, 47-59. doi:10.1016/S0739-7240(03)00044-4

Hennessy, M.B., Kaiser, S., Sachser, N., 2009. Social buffering of the stress response: Diversity, mechanisms, and functions. Front. Neuroendocrinol. 30, 470-482.

doi:10.1016/j.yfrne.2009.06.001

Hofer, H., East, M.L., 2012. Stress and immunosuppression as factors in the decline and extinction of wildlife populations: concepts, evidence, and challenges, in: Aguirre, A., Ostfeld, R., P, D. (Eds.), New Directions in Conservation Medicine: Applied Cases of Ecological Health. Oxford University Press, pp. 82-107.

Hofer, H., East, M.L., 1995. Population dynamics, population size, and the commuting system of Serengeti spotted hyenas, in: Sinclair, A.R.E., Arcese, P. (Eds.), Serengeti II: Dynamics, Management, and Conservation of an Ecosystem. University of Chicago Press, Chicago, pp. 332363. 
Hofer, H., East, M.L., 1993. The commuting system of Serengeti spotted hyaenas: how a predator copes with migratory prey. II. Intrusion pressure and commuters' space use. Anim. Behav. 46, 559-574. doi:10.1006/anbe.1993.1223

Houston, A.I., Stephens, P.A., Boyd, I.L., Harding, K.C., McNamara, J.M., 2007. Capital or income breeding? A theoretical model of female reproductive strategies. Behav. Ecol. 18, 241-250. doi:10.1093/beheco/arl080

IUCN, 2017. Equus quagga (Burchell's zebra) [WWW Document]. URL http://www.iucnredlist.org/details/41013/0 (accessed 6.6.17).

Jeanniard du Dot, T., Rosen, D.A.S., Richmond, J.P., Kitaysky, A.S., Zinn, S.A., Trites, A.W., 2009. Changes in glucocorticoids, IGF-I and thyroid hormones as indicators of nutritional stress and subsequent refeeding in Steller sea lions (Eumetopias jubatus). Comp. Biochem. Physiol. Part A Mol. Integr. Physiol. 152, 524-534. doi:10.1016/j.cbpa.2008.12.010

Kersey, D.C., Dehnhard, M., 2014. The use of noninvasive and minimally invasive methods in endocrinology for threatened mammalian species conservation. Gen. Comp. Endocrinol. 203, 296-306. doi:10.1016/j.ygcen.2014.04.022

Kingdon, J., 1979. Horses, zebras, asses: Equus, in: East African Mammals: An Atlas of Evolution in Africa. Large Mammals. Vol 3. Academic Press London, UK, pp. 124-179.

Kitaysky, A.S., Piatt, J.F., Hatch, S.A., Kitaiskaia, E. V., Benowitz-Fredericks, Z.M., Shultz, M.T., Wingfield, J.C., 2010. Food availability and population processes: severity of nutritional stress during reproduction predicts survival of long-lived seabirds. Funct. Ecol. 24, 625-637. doi:10.1111/j.1365-2435.2009.01679.x

Klingel, H., 2013. Equus quagga Plains zebra (Common zebra), in: Kingdon, J., Happold, D., Butynski, T., Hoffmann, M., Happold, M., Kalina, J. (Eds.), Mammals of Africa. Bloomsbury Natural History, pp. 428-437.

Klingel, H., 2010. Soziale Organisation und Verhalten freilebender Steppenzebras. Z. Tierpsychol. 24, 580-624. doi:10.1111/j.1439-0310.1967.tb00807.x

Klingel, H., 1974. A comparison of the social behaviour of the Equidae., in: Geist, V., Walther, F. (Eds.), The Behaviour of Ungulates and Its Relation to Management. International Union for the Conservation of Nature and Natural Resources, Morges, pp. 124-132.

Klingel, H., 1972. Social behaviour of African equidae. Zool. Africana 7, 175-185. doi:10.1080/00445096.1972.11447438

Klingel, H., 1969a. Reproduction in the plains zebra, equus burchelli boehmi, behaviour and ecological factors. J. Reprod. Fertil. 6, 339-345.

Klingel, H., 1969b. The social organisation and population ecology of the plains zebra (Equus quagga). Zool. Africana 4, 249-263. doi:10.1080/00445096.1969.11447374

Kruuk, H., 1972. The spotted hyeana. University of Chicago Press, Chicago.

Landete-Castillejos, T., Garcia, A., Gómez, J.A., Laborda, J., 2002. Effects of nutritional stress during lactation on immunity costs and indices of future reproduction in Iberian red deer (Cervus elaphus hispanicus). Biol. Reprod. 67, 1613-1620. doi:10.1095/biolreprod.102.004507

Linklater, W.., 2000. Adaptive explanation in socio-ecology: lessons from the equidae. Biol. Rev. 75, 1-20. doi:10.1111/j.1469-185X.1999.tb00039.x

MacNaughton, S., Banyikwa, F., 1995. Plant communities and herbivory, in: Sinclair, A.R.E., Arcese, P. 
(Eds.), Serengeti II: Dynamics, Management and Conservation of an Ecosystem. The University of Chicago Press.

Maddock, L., 1979. The migration and grazing succession, in: Sinclair, A., Norton-Griffiths, M. (Eds.), Serengeti: Dynamics of an Ecosystem. The University of Chicago Press.

Martin, R.D., 1984. Scaling effects and adaptive strategies in mammalian lactation. Symp. Proc. Zool. Soc. London.

McDonnell, S.M., Murray, S.C., 1995. Bachelor and harem stallion behavior and endocrinology. Biol Reprod Mono 1, 577-590.

McEwen, B.S., Wingfield, J.C., 2003. The concept of allostasis in biology and biomedicine. Horm. Behav. 43, 2-15. doi:10.1016/S0018-506X(02)00024-7

McNaughton, S.J., 1990. Mineral nutrition and seasonal movements of African migratory ungulates. Nature 345, 613-615. doi:10.1016/0021-9797(80)90501-9

McNaughton, S.J., 1985. Ecology of a grazing ecosystem: the Serengeti. Ecol. Monogr. 55, 259-294. doi:10.2307/1942578

Mooring, M.S., Patton, M.L., Lance, V.A., Hall, B.M., Schaad, E.W., Fetter, G.A., Fortin, S.S., McPeak, K.M., 2006. Glucocorticoids of bison bulls in relation to social status. Horm. Behav. 49, 369-375. doi:10.1016/j.yhbeh.2005.08.008

Navarro, D.J., 2015. Learning statistics with R: A tutorial for psychology students and other beginners. University of Adelaide.

Ndlela, L.L., Schmidt, S., 2016. Evaluation of wild herbivore faeces from South Africa as a potential source of hydrolytically active microorganisms. Springerplus 5. doi:10.1186/s40064-016-1739-y

Nelson, R.J., Demas, G.E., Klein, S.L., Kriegsfeld, L.J., 2002. Seasonal Patterns of Stress, Immune Function, and Disease. Cambridge University Press, New York.

Norton-Griffiths, M., Herlocker, D., Pennycuick, L., 1975. The patterns of rainfall in the Serengeti ecosystem, Tanzania. East African Wildl. J. 13, 347-374.

Nuñez, C.M.V., Adelman, J.S., Smith, J., Gesquiere, L.R., Rubenstein, D.I., 2014. Linking social environment and stress physiology in feral mares (Equus caballus): Group transfers elevate fecal cortisol levels. Gen. Comp. Endocrinol. 196, 26-33. doi:10.1016/j.ygcen.2013.11.012

Pavitt, A.T., Walling, C.A., Möstl, E., Pemberton, J.M., Kruuk, L.E.B., 2015. Cortisol but not testosterone is repeatable and varies with reproductive effort in wild red deer stags. Gen. Comp. Endocrinol. 222, 62-68. doi:10.1016/j.ygcen.2015.07.009

Périquet, S., Richardson, P., Cameron, E.Z., Ganswindt, A., Belton, L., Loubser, E., Dalerum, F., 2017. Effects of lions on behaviour and endocrine stress in plains zebras. Ethology 1-8. doi:10.1111/eth.12638

Phuc Le, P., Friedman, J.R., Schug, J., Brestelli, J.E., Parker, J.B., Bochkis, I.M., Kaestner, K.H., 2005. Glucocorticoid receptor-dependent gene regulatory networks. PLoS Genet. 1, e16. doi:10.1371/journal.pgen.0010016

Pribbenow, S., Jewgenow, K., Vargas, A., Serra, R., Naidenko, S., Dehnhard, M., 2014. Validation of an enzyme immunoassay for the measurement of faecal glucocorticoid metabolites in Eurasian (Lynx lynx) and Iberian lynx (Lynx pardinus). Gen. Comp. Endocrinol. 206, 166-177. doi:10.1016/j.ygcen.2014.07.015 
R Development Core Team, 2016. R: A Language and Environment for Statistical Computing. Vienna, Austria.

Ransom, J.I., Kaczensky, P. (Eds.), 2016. Wild Equids: Ecology, Management, and Conservation. Johns Hopkins University Press, Baltimore, Maryland.

Rogovin, K., Randall, J.A., Kolosova, I., Moshkin, M., 2003. Social correlates of stress in adult males of the great gerbil, Rhombomys opimus, in years of high and low population densities. Horm. Behav. 43, 132-139. doi:10.1016/S0018-506X(02)00028-4

Romero, L.M., Dickens, M.J., Cyr, N.E., 2009. The reactive scope model - A new model integrating homeostasis, allostasis, and stress. Horm. Behav. 55, 375-389. doi:10.1016/j.yhbeh.2008.12.009

Rubenstein, D.I., 1986. Ecology and sociality in horses and zebras, in: Rubenstein, D., Wrangham, R. (Eds.), Ecological Aspects of Social Evolution. Princeton University Press, Princeton, NJ, pp. 282302.

Rubenstein, D.I., 1978. On predation, competition, and the advantages of group living, in: Bateson, P.P.G., Klopfer, P.H. (Eds.), Perspectives in Ethology, Vol. 3. Springer US, Boston, MA, pp. 205231. doi:10.1007/978-1-4684-2901-5_9

Rubenstein, D.R., Shen, S.-F., 2009. Reproductive conflict and the costs of social status in cooperatively breeding vertebrates. Am. Nat. 173, 650-61. doi:10.1086/597606

Schaller, G.B., 1972. The Serengeti Lion. The University of Chicago Press.

Schmidt, A., Möstl, E., Wehnert, C., Aurich, J., Müller, J., Aurich, C., 2010. Cortisol release and heart rate variability in horses during road transport. Horm. Behav. 57, 209-215. doi:10.1016/j.yhbeh.2009.11.003

Schwarzenberger, F., 2007. The many uses of non-invasive faecal steroid monitoring in zoo and wildlife species 52-74. doi:10.1111/j.1748-1090.2007.00017.x

Sheriff, M.J., McMahon, E.K., Krebs, C.J., Boonstra, R., 2015. Predator-induced maternal stress and population demography in snowshoe hares: the more severe the risk, the longer the generational effect. J. Zool. 296, 305-310. doi:10.1111/jzo.12249

Smuts, G.L., 1976. Reproduction in the zebra mare Equus burchelli antiquorum from the Kruger National Park. Koedoe 19, 89-132. doi:10.4102/koedoe.v19i1.1186

Stearns, S.C., 1992. The evolution of life histories. Oxford University Press.

Taylor, R.J., 1979. The value of clumping to prey when detectability increases with group size. Am. Nat. 113, 299-301. doi:10.1086/283387

Touma, C., Palme, R., 2005. Measuring fecal glucocorticoid metabolites in mammals and birds: the importance of validation. Ann. N. Y. Acad. Sci. 1046, 54-74. doi:10.1196/annals.1343.006

Tung, J., Barreiro, L.B., Johnson, Z.P., Hansen, K.D., Michopoulos, V., Toufexis, D., Michelini, K., Wilson, M.E., Gilad, Y., 2012. Social environment is associated with gene regulatory variation in the rhesus macaque immune system. Proc. Natl. Acad. Sci. 109, 6490-6495. doi:10.1073/pnas.1202734109

Wackernagel, H., 1965. Grant's zebra, Equus burchelli boehmi, at Basel Zoo - a contribution to breeding biology. Int. Zoo Yearb. 5, 38-41. doi:10.1111/j.1748-1090.1965.tb01567.x

Wolf, T.E., Bennett, N.C., Burroughs, R., Ganswindt, A., 2018. The impact of age-class and social 
context on fecal glucocorticoid metabolite levels in free-ranging male giraffes. Gen. Comp. Endocrinol. 255, 26-31. doi:10.1016/j.ygcen.2017.09.022

Woodall, P.F., Skinner, J.D., 1993. Dimensions of the intestine, diet and faecal water loss in some African antelope. J. Zool. 229, 457-471. doi:10.1111/j.1469-7998.1993.tb02648.x

Yarnell, K., Hall, C., Royle, C., Walker, S.L., 2015. Domesticated horses differ in their behavioural and physiological responses to isolated and group housing. Physiol. Behav. 143, 51-57. doi:10.1016/j.physbeh.2015.02.040

York, C. a, Schulte, B. a, 2014. The relationship of dominance, reproductive state and stress in female horses (Equus caballus). Behav. Processes 107, 15-21. doi:10.1016/j.beproc.2014.07.005 